\title{
Low Temperature Tempering of a Medium Carbon Steel in High Magnetic Field
}

\author{
Yudong ZHANG, ${ }^{1,2)}$ Xiang ZHAO, ${ }^{1)}$ Nathalie BOZZOLO, ${ }^{2)}$ Changshu $\mathrm{HE}^{1)}$ Liang ZUO ${ }^{1)}$ and Claude ESLING ${ }^{2)}$ \\ 1) Key Laboratory of Electromagnetic Processing of Materials (Ministry of Education), Northeastern University, Shenyang \\ 110004, P. R. China. $\quad$ 2) LETAM, CNRS-UMR 7078, University of Metz, lle du Saulcy, 57045 Metz Cedex 01, France.
}

(Received on October 28, 2004; accepted on March 31, 2005)

\begin{abstract}
The effect of low temperature magnetic tempering on carbide precipitation in a medium carbon steel, $42 \mathrm{CrMo}$, has been investigated. As-quenched steel specimens were tempered at $200^{\circ} \mathrm{C}$ for 60 min without and with a 14-T magnetic field. Results show that under the magnetic field processing there forms the relatively high-temperature monoclinic $\chi-\mathrm{Fe}_{5} \mathrm{C}_{2}$ carbide with a denser distribution and smaller sizes, as compared to the usual orthorhombic $\eta$ - $\mathrm{Fe}_{2} \mathrm{C}$ carbide obtained without the magnetic field. The impact of the external magnetic field refers to a change in the precipitation sequence of the transition carbides by effectively lowering the Gibbs free energy of the high magnetization phases. The denser distribution and smaller size of $\chi-\mathrm{Fe}_{5} \mathrm{C}_{2}$ precipitates are attributed to the increased nucleation rate and the weaker diffusion capacity required for growth as the formation temperature is lower. This offers additional dispersion strengthening to compensate for the decrease in strength and hardness due to the loss of supersaturation of carbon atoms in the matrix and raises the toughness of the steel.
\end{abstract}

KEY WORDS: Iow temperature tempering; magnetic field; transition carbides; orientation relationship; medium carbon steel.

\section{Introduction}

As many properties of materials are determined by their microstructures, the performance optimization through microstructure modification and control - to meet the ever-increasing demands imposed by almost all branches of industry-has become one of the most challenging subjects in the field of materials science and engineering. Traditional processing methods have become less efficient in the face of these new requirements and gradually lost their dominant status. As a consequence, the introduction of new techniques to materials processing has become a major objective. Recently, the electromagnetic processing of materials (EPM) has emerged as a key procedure and aroused wide attention among materials researchers, especially since various high magnetic fields have been applied to conventional heat treatment processes.

So far, the researches conducted on this topic have focused on the effects of magnetic field on martensitic transformations ${ }^{1-4)}$ and high-temperature diffusional phase transformations between austenite and ferrite..$^{5-12)}$ Some reports deal with the precipitation behaviors of transition carbides.
As most transition carbides are ferromagnetic with various magnetization degrees, the introduction of magnetic field can effectively change their stability by altering their Gibbs free energy levels and thus affect their precipitating courses and behaviors, and in consequence, the final material properties could be changed and controlled. Insight into this aspect is also helpful to gain a better understanding of the complex precipitation processes and the behavior of transition carbides and is of significance from both theoretical and technical points of view.

In the present work, a medium carbon steel was tempered at $200^{\circ} \mathrm{C}$ for $60 \mathrm{~min}$ without and with a 14-T magnetic field, after being quenched in a traditional way. The effects of the magnetic field on the course and behavior of carbide precipitation and its subsequent influences on the mechanical properties of the material have been investigated.

\section{Experimental}

The chemical composition of the medium carbon steel used in this study is given in Table 1. Specimens of dimensions $55 \mathrm{~mm} \times 10 \mathrm{~mm} \times 2 \mathrm{~mm}$ were cut from a hot-rolled rod

Table 1. Chemical composition of $42 \mathrm{CrMo}$ steel (wt.\%).

\begin{tabular}{lllllll}
\hline $\mathrm{C}$ & $\mathrm{Cr}$ & $\mathrm{Mo}$ & $\mathrm{Si}$ & $\mathrm{Mn}$ & others & $\mathrm{Fe}$ \\
\hline $0.38-0.45$ & $0.90-1.20$ & $0.15-0.25$ & $0.20-0.40$ & $0.50-0.80$ & $\mathrm{P} \leq 0.04$ & Bal. \\
& & & & & $\mathrm{S} \leq 0.04$ & \\
& & & & & $\mathrm{Cu} \leq 0.30$ & \\
\hline
\end{tabular}


with their longitudinal direction parallel to the hot-rolling direction. They were then all heated up to $860^{\circ} \mathrm{C}$ and maintained for $20 \mathrm{~min}$, followed by water quenching to obtain a martensite microstructure for subsequent tempering. Both the magnetic and non-magnetic tempering were carried out at $200^{\circ} \mathrm{C}$ for 15,30 and $60 \mathrm{~min}$ and $150^{\circ} \mathrm{C}$ for $60 \mathrm{~min}$ in the same furnace installed in a $15-\mathrm{T}$ cryocooled superconducting magnet of $52 \mathrm{~mm}$ in bore size. The specimens were placed in the central (zero magnetic force) region with their longitudinal direction parallel to the magnetic field direction. In the case of magnetic tempering, a 14-T high magnetic field was applied during the whole heating and cooling processes.

The specimens tempered were further cut out along their longitudinal direction for the SEM and TEM observations and for the mechanical tests of hardness, strength and impact toughness. Non-standard tensile and Charpy impact specimens were used, as shown in Fig. 1. The morphologies of precipitates and the orientation relationships between precipitates and matrix were examined by using a JEOL JSM-6500F SEM and a Philips CM $200 \mathrm{LaB}_{6}$ cathode TEM, respectively. Vicker's hardness values were measured with a $5 \mathrm{~kg}$ load. Tensile tests were carried out on a Shimadzu AG-5000A unit with a load of $2500 \mathrm{~kg}$, and Charpy impact tests on a JB-5 Charpy impact machine with a maximum measurement range of $29.4 \mathrm{~J}$. The fracture morphologies of the tensile and impact specimens were also ob-

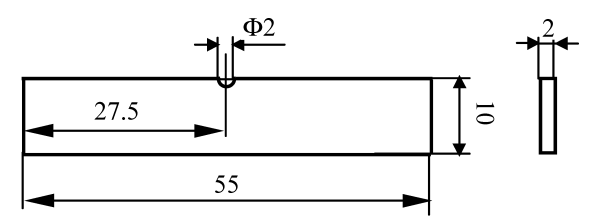

(a) Charpy impact specimen

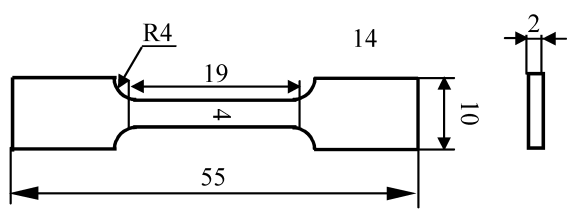

(b) Tensile specimen

Fig. 1. Illustration of tensile and Charpy specimens.

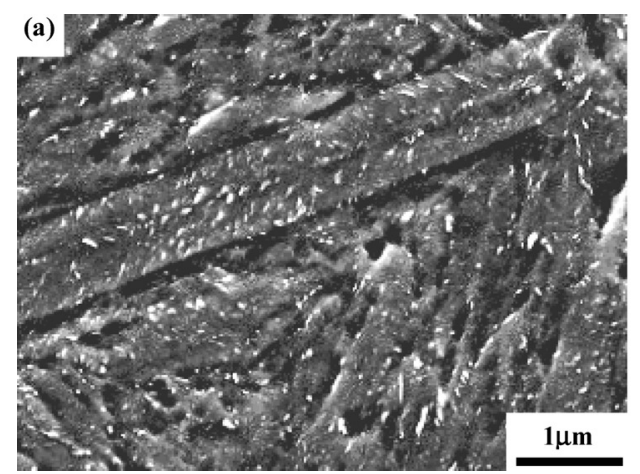

served by SEM.

\section{Results}

Figure 2 (TEM bright field micrograph) shows the initial microstructure of the as-quenched specimen, which is characteristic of $\alpha^{\prime}$ martensite plates. For the specimens tempered at $200^{\circ} \mathrm{C}$ for $60 \mathrm{~min}$, without and with an applied 14$\mathrm{T}$ high magnetic field, the carbides precipitated within the martensite plates (Fig. 3, SEM micrographs). In both cases, the precipitates appeared in the form of thin platelets arranged in parallel to some directions within the matrix. However, the imposition of a magnetic field during tempering has yielded more densely distributed platelets with a smaller average length, as compared with the non-magnetic tempering case. SEM observation showed that when tempered at $200^{\circ} \mathrm{C}$ with different times $(15,30$ and $60 \mathrm{~min})$, precipitation occurs at very early stage. The amount and size of the precipitates increase with the increase of the tempering time. No carbide precipitation was observed in the specimens tempered at $150^{\circ} \mathrm{C}$ for 60 min either without or with the magnetic field.

The crystal structure of precipitates and their orientation relations to the matrix were identified by electron diffraction in TEM. The carbide formed during the non-magnetic tempering is of the typical orthorhombic $\eta$-Fe ${ }_{2} \mathrm{C}$ type and is correlated to tempered martensite $\alpha^{\prime \prime}$ by (110) $\alpha^{\prime \prime} / /(200) \eta$ and $[1 \overline{1} \overline{3}] \alpha^{\prime \prime} / /[0 \overline{2} 0] \eta$ (Fig. 4(a)). However, the precipitated carbide in the magnetic field is referred to as the monoclinic $\chi-\mathrm{Fe}_{5} \mathrm{C}_{2}$ type with the orientation correlation defined by $(01 \overline{1}) \alpha^{\prime \prime} / /(021) \chi$ and $[1 \overline{3} \overline{3}] \alpha^{\prime \prime} / /[5 \overline{3} 6] \chi$ (Fig. 4(b)).

The mechanical properties of the as-tempered specimens

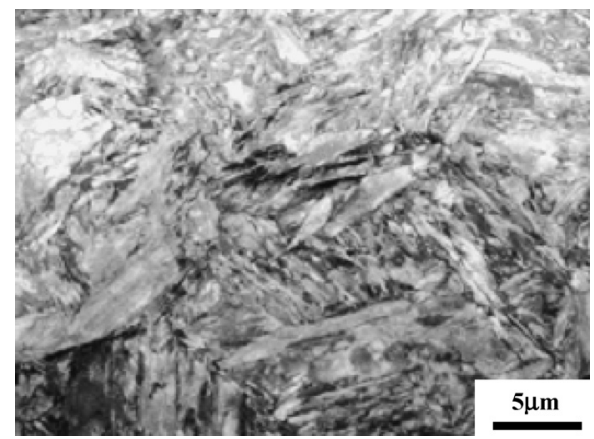

Fig. 2. Bright-field TEM micrograph of initial martensite in asquenched specimen.

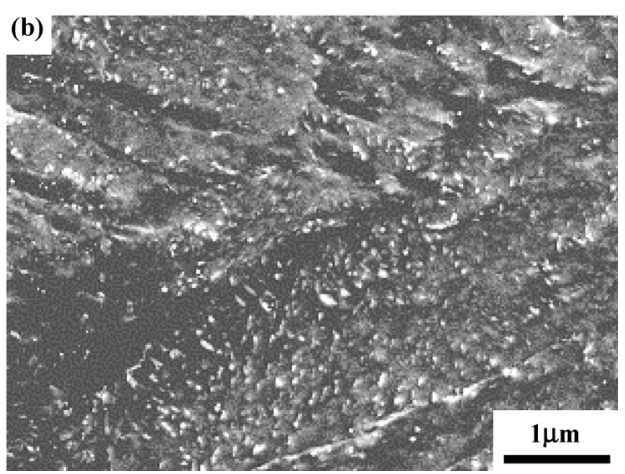

Fig. 3. SEM secondary electron images of carbide precipitates (bright areas) within martensite in specimens tempered at $200^{\circ} \mathrm{C}$ for $60 \mathrm{~min}$ (a) without and (b) with 14-T magnetic field (the magnetic-field direction is vertical in the micrograph). 

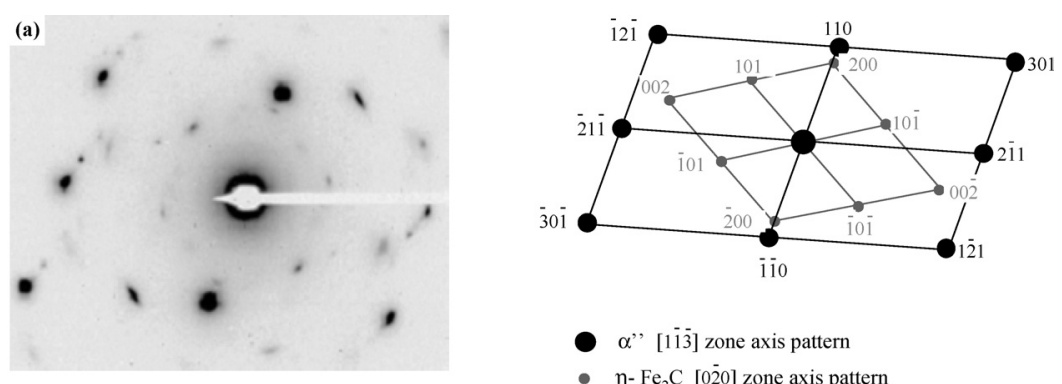

- $\alpha$ " $[1 \overline{1} \overline{3}]$ zone axis pattern

- $\eta-\mathrm{Fe}_{2} \mathrm{C}[020]$ zone axis pattern

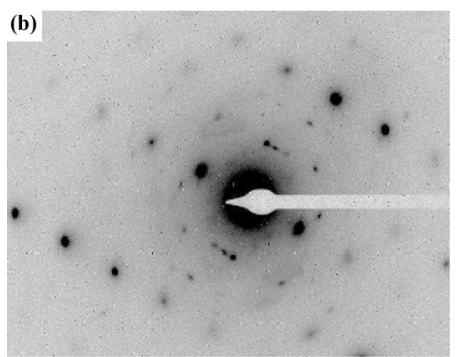

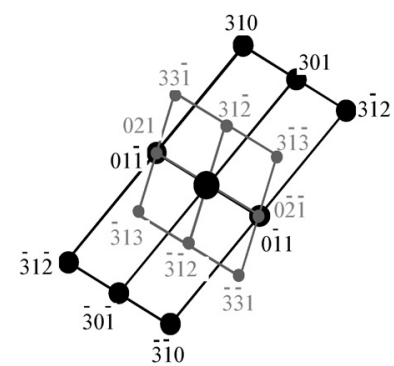

$\alpha,[133]$ zone axis pattern

- $\chi-\mathrm{Fe}_{5} \mathrm{C}_{2}[536]$ zone axis pattern
Fig. 4. Diffraction patterns and corresponding indexing for (a) $\chi-\mathrm{Fe}_{2} \mathrm{C}$ formed during nonmagnetic tempering at $200^{\circ} \mathrm{C}$ for $60 \mathrm{~min}$ and (b) $\chi-\mathrm{Fe}_{5} \mathrm{C}_{2}$ formed during magnetic tempering at $200^{\circ} \mathrm{C}$ for $60 \mathrm{~min}$.

Note: the two patterns are taken with different camera lengths; as a consequence, the same reciprocal spots in the two patterns have different reciprocal vector lengths.

Table 2. Mechanical properties of specimens tempered at $200^{\circ} \mathrm{C}$ for $60 \mathrm{~min}$ without and with $14-\mathrm{T}$ magnetic field.

\begin{tabular}{|cccccc|}
\hline $\begin{array}{c}\text { Magnetic field } \\
(\text { Tesla })\end{array}$ & $\begin{array}{c}\text { Vicker's } \\
\text { hardness }\end{array}$ & $\begin{array}{c}\text { Yield strength } \\
(\mathrm{MPa})\end{array}$ & $\begin{array}{c}\text { Tensile strength } \\
(\mathrm{MPa})\end{array}$ & $\begin{array}{c}\text { Elongation } \\
(\%)\end{array}$ & $\begin{array}{c}\text { Impact toughness } \\
\left(\mathrm{MJ} / \mathrm{m}^{2}\right)\end{array}$ \\
\hline 0 & 570.8 & 1583 & 1850 & 2.0 & 0.767 \\
14 & 552.6 & 1557 & 1877 & 2.1 & 0.838 \\
\hline
\end{tabular}
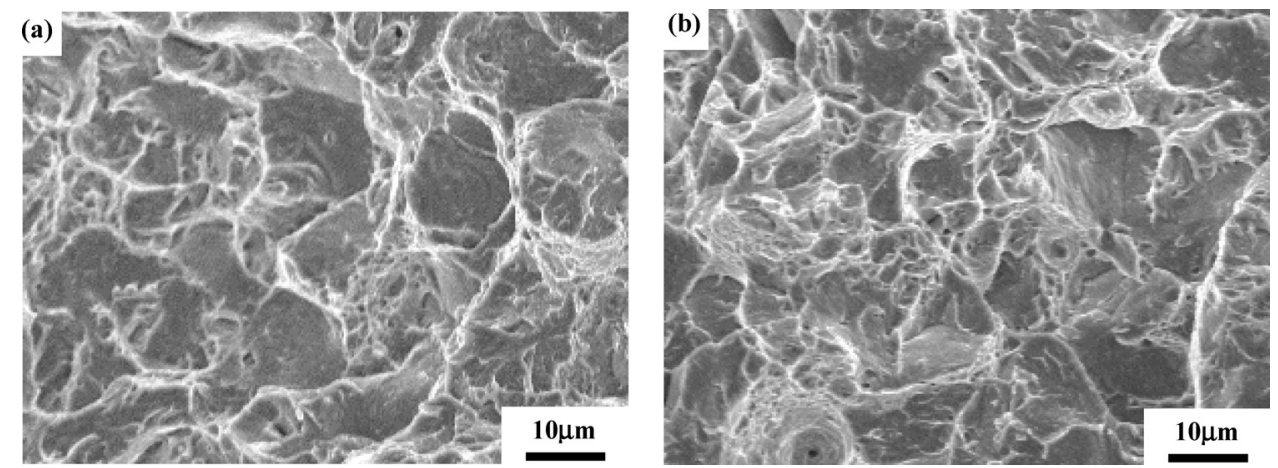

Fig. 5. SEM secondary electron images of impact fractures in specimens tempered at $200^{\circ} \mathrm{C}$ for $60 \mathrm{~min}$ (a) without and (b) with 14-T magnetic field. The fractographs show a mixture of dimples and intergranular cleavages.

are given in Table 2. It was observed that, with the applied magnetic field, the impact toughness of the tested steel was enhanced by $9 \%$ whereas the Vicker's hardness and the yield strength were not changed significantly. The fractures of all the impact specimens were featured with a mixture of dimples and intragranular cleavages (Fig. 5, SEM micrographs). However, the magnetic tempering resulted in a higher ratio of dimples than that of the non-magnetic tempering and the impact toughness was improved. As there was - to some extent - intragranular fracture in both cases, crack propagation along certain planes of tempered martensite and the interfaces between carbide and tempered martensite must have played an important role.

\section{Discussion}

It has been established that a quenched martensite microstructure is highly unstable due to (1) the supersaturation of carbon atoms in the body-centered tetragonal crystal lattice, (2) the strain energy associated with fine dislocation or twin substructure, (3) the interfacial energy associated with high density of lath or plate boundaries and (4) the retained austenite. When it undergoes tempering, the factors causing unstability supply various driving forces for microstructural evolution and finally give rise to property modification. According to Cohen and his coworkers, ${ }^{13-16)}$ Hirotsu and Nagakura, ${ }^{17}$ the transition carbide, either hexagonal $\varepsilon-\mathrm{Fe}_{2} \mathrm{C}$ or orthorhombic $\eta-\mathrm{Fe}_{2} \mathrm{C}$, precipitates from martensite at low tempering temperatures. The driving 
force for the carbide precipitation is supplied by the supersaturation of carbon atoms in the matrix. When the tempering temperature rises, the metastable $\varepsilon-\mathrm{Fe}_{2} \mathrm{C}$ or $\eta-\mathrm{Fe}_{2} \mathrm{C}$ dissolves into the matrix and the monoclinic $\chi-\mathrm{Fe}_{5} \mathrm{C}_{2}$ precipitates. As $\chi-\mathrm{Fe}_{5} \mathrm{C}_{2}$ is also metastable, it turns to $\mathrm{Fe}_{3} \mathrm{C}$ at higher tempering temperatures.

In this work, for the specimens tempered at $150^{\circ} \mathrm{C}$ for $60 \mathrm{~min}$, no precipitation of transition carbides was observed. When tempered at $200^{\circ} \mathrm{C}$ for $60 \mathrm{~min}$, without or with a 14-T magnetic field, carbide precipitated and took the form of $\chi-\mathrm{Fe}_{2} \mathrm{C}$ and $\eta-\mathrm{Fe}_{5} \mathrm{C}_{2}$ respectively. It is interesting to note that, in the magnetic tempering case, $\eta-\mathrm{Fe}_{5} \mathrm{C}_{2}$ precipitated directly from the matrix martensite without the intermediate stage of the $\chi-\mathrm{Fe}_{2} \mathrm{C}$ formation. This would indicate that the application of a magnetic field changes the precipitation sequence of those transition carbides. Although the precipitation occurs at the early stage of the tempering at $200^{\circ} \mathrm{C}$, the tendency that the precipitates increase both in the amount and size shows the progressive formation of the precipitates. Therefore, there is little chance for $\eta-\mathrm{Fe}_{2} \mathrm{C}$ to form and dissolve and then the $\chi$ $\mathrm{Fe}_{5} \mathrm{C}_{2}$ to form in the magnetic field tempered specimens.

From the thermodynamic point of view, $\varepsilon-\mathrm{Fe}_{2} \mathrm{C}, \eta-\mathrm{Fe}_{2} \mathrm{C}$, $\chi-\mathrm{Fe}_{5} \mathrm{C}_{2}$ and $\mathrm{Fe}_{3} \mathrm{C}$ are all ferromagnetic at $200^{\circ} \mathrm{C} .{ }^{18,19)}$ The application of an external magnetic field can lower their Gibbs free energies and thus change their formation sequence. The magnetic property data for $\varepsilon-\mathrm{Fe}_{2} \mathrm{C}$ and $\mathrm{Fe}_{3} \mathrm{C}$ are currently well documented, ${ }^{18)}$ but those for $\eta-\mathrm{Fe}_{2} \mathrm{C}$ and $\chi-\mathrm{Fe}_{5} \mathrm{C}_{2}$ are very modest. In fact, the overall magnetic moments of those iron carbides are determined by the magnetic moments of their iron atoms ${ }^{18)}$ and, for each of them, the magnetic moment originates from the unsaturated spins of its inner electrons of the atom. As the total magnetic moment of an assembly of atoms can be added up to individual ones, ${ }^{20)}$ it can be inferred that the magnetic properties of metallic compounds are mainly determined by their composition and crystal structure. Therefore, metallic compounds with similar composition and structure may be considered as having similar magnetic properties. Indeed, such a similarity in structure exists between $\eta-\mathrm{Fe}_{2} \mathrm{C}$ and $\varepsilon-\mathrm{Fe}_{2} \mathrm{C}^{21)}$ and between $\chi-\mathrm{Fe}_{5} \mathrm{C}_{2}$ and $\mathrm{Fe}_{3} \mathrm{C}$. ${ }^{18)}$

On the basis of the above presumptions, the temperature variations of magnetization of $\eta-\mathrm{Fe}_{2} \mathrm{C}, \chi-\mathrm{Fe}_{5} \mathrm{C}_{2}$ as well as $\alpha$-Fe were calculated by using the Weiss molecular field model, ${ }^{22)}$ as shown in Fig. 6. Since both carbides can be magnetized in a magnetic field to some extent, the Gibbs free energy is lowered in relation to their magnetization. The corresponding energy drop amounts to $-\int \mathrm{B}_{0} \cdot \mathrm{dM}^{23)}$ (where is the induction of the applied magnetic field and stands for the magnetization). For a clear description of different forms of magnetic energy, please see the Appendix. It is seen from Fig. 6 that the magnetization of $\chi-\mathrm{Fe}_{5} \mathrm{C}_{2}$ at $200^{\circ} \mathrm{C}$ is obviously higher than that of $\eta-\mathrm{Fe}_{2} \mathrm{C}$. Therefore, in a 14-T magnetic field, the Gibbs free energy of $\eta-\mathrm{Fe}_{5} \mathrm{C}_{2}$ probably drops beyond that of $\eta-\mathrm{Fe}_{2} \mathrm{C}$, and $\chi-\mathrm{Fe}_{5} \mathrm{C}_{2}$ becomes more stable at this temperature. The change in stability under the magnetic field at this tempering temperature can be illustrated schematically with their Gibbs free energy vs. the carbon concentration curves given in Fig. 7. In consequence, $\chi-\mathrm{Fe}_{5} \mathrm{C}_{2}$ would precipitate before $\eta$ - $\mathrm{Fe}_{2} \mathrm{C}$ while the tempering in the magnetic field.

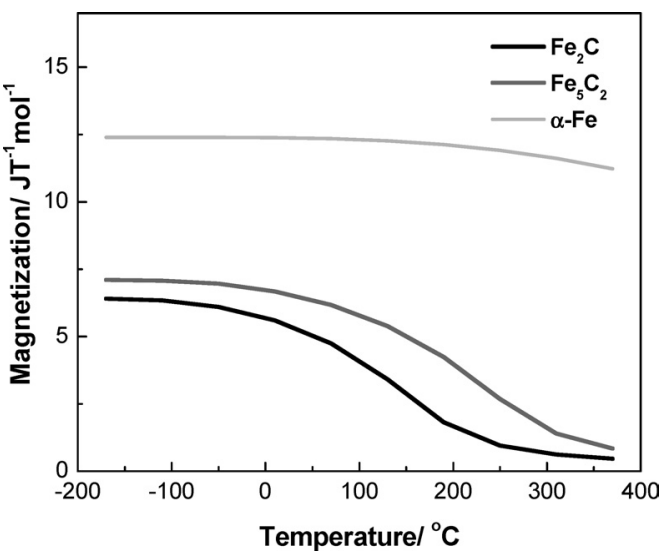

Fig. 6. Variations of magnetization with temperature for $\mathrm{Fe}_{2} \mathrm{C}$, $\mathrm{Fe}_{5} \mathrm{C}_{2}$ and $\alpha$-Fe.

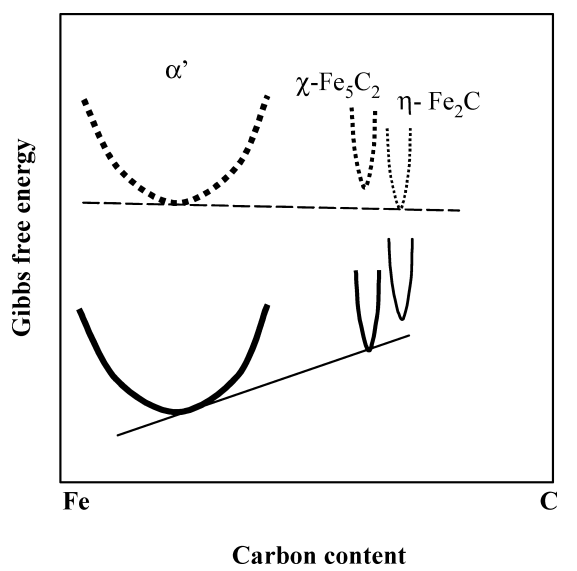

Fig. 7. Schematic diagram of Gibbs free energy $v s$. carbon concentration for $\alpha^{\prime}$ martensite, $\chi-\mathrm{Fe}_{5} \mathrm{C}_{2}$ and $\eta-\mathrm{Fe}_{2} \mathrm{C}$ at $200^{\circ} \mathrm{C}$ without (dash line) or with (solid line) 14-T magnetic field.

As the formation temperature of $\chi-\mathrm{Fe}_{5} \mathrm{C}_{2}$ drops in an external magnetic field, $\chi-\mathrm{Fe}_{5} \mathrm{C}_{2}$ could nucleate at lower temperatures with a higher undercooling degree and increased nucleation rate. Indeed, $\chi-\mathrm{Fe}_{5} \mathrm{C}_{2}$ formed in the 14-T magnetic field displays a denser distribution. Moreover, the applied magnetic field showed some influence on the diffusivity, ${ }^{24)}$ as the sizes of the carbide precipitates are smaller when the magnetic field was applied (Fig. 3(a), 3(b)).

During the $\chi-\mathrm{Fe}_{5} \mathrm{C}_{2}$ carbide formation supersaturated carbon atoms in martensite have to diffuse out, which lead to some change in the mechanical properties of a material. Usually, with the increase of tempering temperature, the toughness and the plasticity of materials rise, whereas the strength and the hardness drop. Though-as appeared in this study on the precipitation of $\chi-\mathrm{Fe}_{5} \mathrm{C}_{2}$ in the magnetic field - the toughness and the plasticity increase, the strength of the material does not drop but remains nearly unchanged, as can be seen from Table 2. This may be attributed to the denser distribution and the smaller size (longitudinally) of the $\chi-\mathrm{Fe}_{5} \mathrm{C}_{2}$ carbides. On the one hand, the denser distribution results in additional dispersion strengthening and compensates for the decrease in strength due to the loss of supersaturation of carbon atoms in the matrix. On the other hand, as the carbide/matrix interfaces are the preferential sites for cracking, the smaller sizes of carbides may effectively hinder the crack propagation. As a result, 
the toughness of the material can be improved.

\section{Conclusions}

When the specimens of a $42 \mathrm{CrMo}$ steel are tempered at $200^{\circ} \mathrm{C}$ for $60 \mathrm{~min}$ in a $14-\mathrm{T}$ magnetic field, the high temperature monoclinic $\chi-\mathrm{Fe}_{5} \mathrm{C}_{2}$ carbide is precipitated with an orientation relationship of $(01) \alpha^{\prime \prime} / /(021) \chi$ and [133] $\alpha^{\prime \prime} / /$ [536] $\chi$. However, when tempering under the same thermal conditions, but without the magnetic field, the precipitates are the common orthorhombic $\eta-\mathrm{Fe}_{2} \mathrm{C}$ having an orientation relationship of $\mathrm{A}$ and $\mathrm{B}$.

Considering that the magnetization of $\chi-\mathrm{Fe}_{5} \mathrm{C}_{2}$ at $200^{\circ} \mathrm{C}$ is higher than that of $\chi-\mathrm{Fe}_{2} \mathrm{C}$ and its Gibbs free energy drops beyond that of $\eta-\mathrm{Fe}_{2} \mathrm{C}$ in a 14-T magnetic field, $\chi$ $\mathrm{Fe}_{5} \mathrm{C}_{2}$ precipitates before $\eta-\mathrm{Fe}_{2} \mathrm{C}$ does. This may indicate that the applied magnetic field has an impact as it changes the precipitation sequence of those carbides.

Since $\chi-\mathrm{Fe}_{5} \mathrm{C}_{2}$ is precipitated at relatively low temperature with high undercooling degree, its nucleation rate is higher than that of $\eta-\mathrm{Fe}_{2} \mathrm{C}$. The denser distribution of $\chi-$ $\mathrm{Fe}_{5} \mathrm{C}_{2}$ produces additional dispersion strengthening, and this compensates for the loss of strength linked to the decrease in carbon supersaturation in the matrix. Parallel to this, the lower formation temperature reduces the mobility of atoms and hence restrains the growth of $\chi-\mathrm{Fe}_{5} \mathrm{C}_{2}$. The final size of $\chi-\mathrm{Fe}_{5} \mathrm{C}_{2}$ precipitates is smaller, which contributes to an increase of the toughness by reducing the area of carbide/matrix interfaces for the crack propagation of the material. Insight into these aspects would contribute to a better understanding of the precipitation procedure of transition carbides under a high magnetic field.

\section{Acknowledgements}

This work was supported by the National Science Fund for Distinguished Young Scholars (Grant No. 50325102), the National Natural Science Foundation of China (Grant No. 50234020) and the National High Technology Research and Development Program of China (Grant No. 2002AA336010). We also gratefully acknowledge the support obtained in the frame of the Chinese-French Cooperative Research Project (PRA MX00-03) and the Key International Science and Technology Cooperation Program (Grant No. 2003DF010007). The authors would like to thank the High Magnetic Field Laboratory for Superconducting Materials, Institute for Materials Research, Tohoku University, for the access to the magnetic field experiments.

\section{REFERENCES}

1) M. A. Krivoglaz and V. D. Sadovskiy: Fiz. Met. Metalloved., 18 (1964), 502.

2) M. L. Bernshteyn, G. I. Granik and P. R. Dolzhanskiy: Fiz. Met. Metalloved., 19 (1965), 882.

3) T. Kakeshita, K. Shimizu, T. Maki, I. Tamura, S. Kijima and M. Date: Scr. Metall., 19 (1985), 973.

4) T. Kakeshita, T. Fukuda, T. Saburi, K. Kindo and S. Endo: Physica $B, 237-238$ (1997), 603.

5) M. Shimotomai and K. Maruta: Scr. Mater, 42 (2000), 499.

6) M. Enomoto, H. Guo, Y. Tazuke, Y. R. Abe and M. Shimotomai: Metall. Mater. Trans. A, 32A (2001), 445.

7) M. Shimotomai, K. Maruta, K. Mine and M. Matsui: Acta Mater., 51 (2003), 2921
8) H. Guo and M. Enomoto: Mater. Trans. JIM, 41 (2000), 911.

9) H. D. Joo, J. K. Choi, S. U. Kim, N. S. Shin and Y. M. Koo: Metall. Mater. Trans. A, 35A (2004), 1663.

10) J. K. Choi, H. Ohtsuka, Y. Xu and W. Y. Choo: Scr. Mater, 43 (2000), 221.

11) H. Ohtsuka, Y. Xu and H. Wada: Mater. Trans. JIM, 41 (2000), 907.

12) H.-J. Hao and H. Ohtsuka: Mater. Trans., 45 (2004), 2622.

13) C. S. Roberts, B. L. Averbach and M. Cohen: Trans. ASM, 45 (1953), 576.

14) B. S. Lement, B. L. Averbach and M. Cohen: Trans. ASM, 46 (1954), 851.

15) F. E. Werner, B. L. Averbach and M. Cohen: Trans. ASM, 49 (1957), 823.

16) B. S. Lement, B. L. Averbach and M. Cohen: Trans. ASM, 47 (1955), 291.

17) Y. Hirotsu and S. Nagakura: Acta Metall., 20 (1972), 645.

18) D. Fruchart, R. Fruchart, Ph. L'Heritier, K. Kanematsu, R. Madar, S. Misawa, Y. Nakamura, P. J. Webster and K. R. A. Ziebeck: Alloys and Compounds of d-Elements with Main Group Elements, in: Magnetic Properties of Metals, ed. by H. P. J. Wijn, LandoltBörnstein, Vol. 19c, Springer-Verlag, Berlin, (1988), 24

19) Z. D. Zhang, R. Kershaw, K. Dwight and A. Wold: DODXA (Techical Report), 12 May, (1986), 8.

20) J. Crangle: The Magnetic Properties of Solids, Edward Arnold (Publisher) Ltd., London, (1977), 26

21) G. Krauss: Principles of Heat Treatment of Steel, American Society for Metals, Ohio, (1980), 200.

22) D. Jile: Introduction to Magnetism and Magnetic Materials, Chapman \& Hall, London, (1991), 259.

23) E. du Trémolet de Lacheisserie: Magnétisme-Fondements, Vol. 1, Grenoble Sciences, Grenoble, (2000), 68.

24) S. Tsurekawa and T. Watanabe: CAMP-ISIJ, 17 (2004), 1210.

\section{Appendix}

Magnetism is a complicated issue and there exist several forms of magnetic energy, of different origins. Different references give various expressions, but in most of those dealing with the phase transformations under magnetic field, the free energies from field are simply expressed as $M H$ or $\int_{0}^{H} M d H$ and there is no detailed definition, nor a description of its origin. It is surely helpful to provide a detailed definition of the various magnetic energies, based on the clear presentation in the reference. ${ }^{23)}$ The authors distinguish three types of energy for a magnetized material set in an applied field. The demagnetization energy is defined as the work required to overcome the repulsion between magnetic domains when the material is magnetized to align these domains parallel to the direction of the applied magnetic field. The density of its volume energy is $1 / 2 \vec{M} \cdot \vec{B}_{\mathrm{d}}\left(B_{\mathrm{d}}\right.$ is the induction of demagnetization field). The Zeeman energy is the work required - the material being already magnetized to a constant magnetization $\mathrm{M}$ - to raise the field from zero to $H_{0}$. The density of its volume energy is $\vec{B}_{0} \cdot \vec{M}$. The sum of the demagnetization energy and the Zeeman energy is called the magnetostatic energy. Finally, the intrinsic magnetization energy is the work provided to the material by raising the level of its magnetization to $M$ with a field $B_{0}$. Its corresponding density of volume energy is $\int_{0}^{H_{0}} \vec{B}_{0} \cdot d \vec{M}$. Obviously only the intrinsic magnetization energy and the demagnetization energy are the energy provided to change the internal energy of the material. As the demagnetization energy is numerically much smaller than the intrinsic magnetization energy, only this latter energy is concerned to determine the influence of the magnetic field applied on the solid-state phase transformations. 